\title{
GROUND-BASED OBSERVATIONS OF PKS2155-304 IN
}

\author{
NOVEMBER 1991
}

\author{
A. BLECHA ${ }^{1}$, T. J.-L. COURVOISIER ${ }^{1}$, H. D. ALLER ${ }^{2}$, M. F. ALLER ${ }^{2}$, \\ P. BOUCHET ${ }^{3}$, P. BRATSCHI ${ }^{1}$, M. T. CARINI ${ }^{4}$, M. DONAHUE $^{5}$, \\ E. D. FEIGELSON ${ }^{6}$, A. V. FILIPPENKO ${ }^{7}$, I. S. GLASS $^{8}$, J. HEIDT ${ }^{9}$, \\ P. A. HUGHES ${ }^{2}$, R. I. KOLLGAARD ${ }^{6}$, T. MATHESON ${ }^{7}$, H. R. MILLER ${ }^{10}$, \\ J. C. NOBLE ${ }^{10}$, P. S. SMITH ${ }^{11}$ and S. WAGNER ${ }^{9}$ \\ ${ }^{1}$ Geneva Observatory, CH-1290 Sauverny, Switzerland \\ ${ }^{2}$ Astronomy Department, University of Michigan, Ann Arbor, Michigan 48109-1090, US \\ ${ }^{3}$ European Southern Observatory, Casilla 19001, Santiago 19, Chil \\ ${ }^{4}$ Computer Sciences Corporation, NASA/GSFC, Greenbelt, MD 20771, US \\ ${ }^{5}$ Carnegie Observatories, 813 Santa Barbara St., Pasadena, CA 91101, US \\ ${ }^{6}$ Department of Astronomy and Astrophysics, 525 Davey Lab, Pennsylvania State University, \\ University Park, PA 16802, USA \\ ${ }^{7}$ Department of Astronomy, University of California, Berkeley, CA 94720, USA \\ ${ }^{8}$ South Africa Astronomical Observatory, PO Box 9, Observatory 7935, South Africa \\ ${ }^{9}$ Landessternwarte Heidelberg-Königstuhl, Königstuhl, D-69117 Heidelberg, Germany \\ ${ }^{10}$ Department of Physics, Georgia State University, Atlanta, GA 30303 \\ ${ }^{11}$ Steward Observatory, University of Arizona, Tucson, AZ 85721, USA
}

\begin{abstract}
We present ground-based data of the BL Lac object PKS 2155-305 obtained during a large international campaign spanning the electro-magnetic spectrum from the radio waves to $\mathrm{X}$-rays in November 1991. For the complete description of the observations and data analysis we refer to the paper by Courvoisier et al. 1993, and references therein. The ground-based data include radio, infrared JHKL and UBVRI fluxes as well as optical and near IR polarimetry.

The broad-band optical and near IR data from U to I exhibit the same behaviour in all bands: the flux nearly doubled over the well-covered period of 23 days. The cross-correlation function does not reveal any significant changes in the light-curves. Though significant variations in 24 hours have been recorded, the cumulated Fourier power spectrum drops to the noise level for periods shorter than 2.5 days. The spectral index remained constant.

The polarised flux varied by a larger factor than the total flux and did not follow the same pattern. The degree of polarisation and polarisation angle are nearly independent of the wavelength and are strongly correlated in all filters.

In the radio domain the spectral index increased from -0.1 on November 5 to +0.02 on 25 -th.

The absence of the lag between the optical and infrared bands and the polarisation variations are consistent with a model in which the variability is caused by micro-lensing of the source (Stickel, Fried and Kühr 1988). One would, however, expect in this model that the variation in the polarisation and the total flux are tightly correlated contrary to what is observed.

The constant shape of the continuum spectral energy suggests that only the number of electrons whose emission is beamed towards the observer changes, rather than the arrival of fresh electrons that are being accelerated.

The variability of the polarisation may be explained by changes in the geometry of the magnetic field (dominant direction). This is consistent with the observed variations of the polarisation angle.
\end{abstract}

\section{References}

Courvoisier T. J.-L. et al. 1993 Ap. J. submitted

Stickel M., Fried J.W. and Kühr H., 1988, A\&A 191, L16

319

T. J.-L. Courvoisier and A. Blecha: Multi-Wavelength Continuum Emission of AGN, 319.

(C) 1994 IAU. Printed in the Netherlands. 